IN BRIEF

- Assesses the degree of contamination of endodontic files after reprocessing in a number of general dental practices.

- Each file was assessed for visual contamination and residual blood deposits.

- Seventy-five per cent of files showed visible evidence of contamination and $7 \%$ of files had residual blood deposits.

- Endodontic files are not reliably decontaminated using procedures readily available in general dental practice.

\title{
A study of visual and blood contamination on reprocessed endodontic files from general dental practice
}

\author{
S. Letters, ${ }^{1}$ A. J. Smith, ${ }_{1}^{2}$ S. McHugh ${ }^{3}$ and J. Bagg ${ }^{4}$
}

Objective This study examined methods used for reprocessing endodontic instruments in general dental practice and determined the degree of residual visual contamination and blood contamination on 250 reprocessed files collected from 25 general dental practices.

Materials and methods A questionnaire was administered to 25 general dental practitioners to obtain information on the re-processing of used endodontic files. Ten files which had been used and reprocessed were also collected from each practice. These were examined visually under a dissecting light microscope for residual contamination and then tested for blood deposits using the Kastle-Meyer test.

Results Nineteen of the 25 practices used stainless steel hand files. No practitioners used endodontic files as single use devices. Ninety-two per cent of the practitioners discarded and replaced files when they were bent or damaged. Several decontamination methods were reported. The two combinations employed most frequently were manual cleaning and autoclaving or manual cleaning, followed by ultrasonic cleaning and autoclaving. Of the 250 files, $75 \%$ showed some degree of visual contamination and seven percent tested positive for residual blood. Blood contaminated files were significantly more heavily contaminated when examined visually. Large variations were found in residual contamination of files collected from practices using the same methods of decontamination.

Conclusions While all practitioners re-used endodontic files, the variations in decontamination methods reported indicate a lack of clarity on best practice. This study demonstrates that endodontic files are not reliably decontaminated by methods currently employed in dental practice.

\section{INTRODUCTION}

During recent years both patients and the media have demonstrated a heightened awareness of infection control issues in dentistry. Effective sterilisation of all re-usable instruments and

${ }^{1}$ Visiting General Dental Practitioner, ${ }^{2 *}$ Senior Lecturer in Oral Microbiology, ${ }^{3}$ Statistician, ${ }^{4}$ Professor of Clinical Microbiology, University of Glasgow Dental School, 378 Sauchiehall Street, Glasgow G2 3JZ

*Correspondence to: Dr Andrew Smith, Infection Research Group, Level 9, University of Glasgow Dental School, 378 Sauchiehall Street, Glasgow G2 3JZ

Email:a.smith@dental.gla.ac.uk

\section{Refereed paper}

Accepted 10 January 05

doi: 10.1038/sj.bdj.4812811

๑ British Dental Journal 2005; 199: 522-525 disinfection of surfaces within the surgery are key elements of the strategy to minimise potential risk of transmission of infections. ${ }^{1}$ For both of these procedures to be effective, thorough cleaning is an essential pre-requisite.

The emergence of variant CJD (vCJD) has provided cross-infection control with a new challenge. For vCJD, unlike other forms of CJD, abnormal prion protein is found in lymphoreticular tissue, including tonsil and appendix, as well as in the central nervous system (CNS). Prion protein accumulation has also been detected in the trigeminal ganglion, raising the possibility that distal branches of the trigeminal nerve may harbour infectivity, although a study of oral tissues from three patients who had died of vCJD has failed to demonstrate the presence of abnormal prion proteins using immunohistochemical techniques. ${ }^{2}$ More recent work using very sensitive immunochemical assays has demonstrated the presence of abnormal prions in muscle tissue in cases of sporadic CJD. ${ }^{3}$ These more recent findings demonstrate the need for prudence when considering measures to reduce the potential for iatrogenic transmission of human transmissible spongiform encephalopathies (TSEs).

Abnormal prion proteins adhere to surfaces such as stainless steel and are resistant to many methods of decontamination. ${ }^{4}$ There is a potential for onward transmission of vCJD via re-usable medical devices ${ }^{5}$ and the large volume of instruments used in dental surgery has raised the theoretical possibility of transmission in that setting. ${ }^{6}$

In a recent survey of decontamination practices employed by UK general dental practitioners, 88\% reprocessed endodontic files after use. ${ }^{7}$ These figures are of concern if endodontic files cannot be reliably cleaned and decontaminated. Smith and co-workers reported that $76 \%$ of files from a sample of general dental practices showed visible surface debris when examined under magnification following cleaning and sterilisation procedures. ${ }^{8}$

The study reported here extends a previous study ${ }^{8}$ by obtaining endodontic files from different dental practices, obtaining data on methods currently used for reprocessing these devices and determining the degree of blood contamination on reprocessed files collected from 25 general dental practices.

\section{MATERIALS AND METHODS}

A random sample of 25 general dental practices was identified from the GRID group (Glasgow Research in Dental Practice). Each practice was informed of the nature of the survey and 


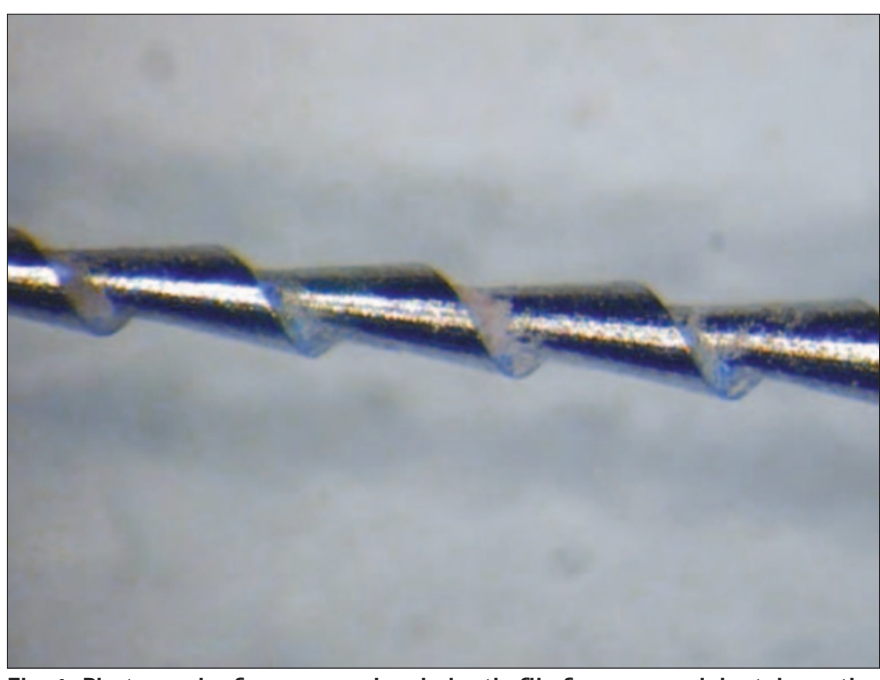

Fig. 1 Photograph of reprocessed endodontic file from general dental practice decontaminated and ready for re-use. (File size 20. Magnification X 50. Decontamination method used was manual cleaning, ultrasonicating and steam sterilisation).

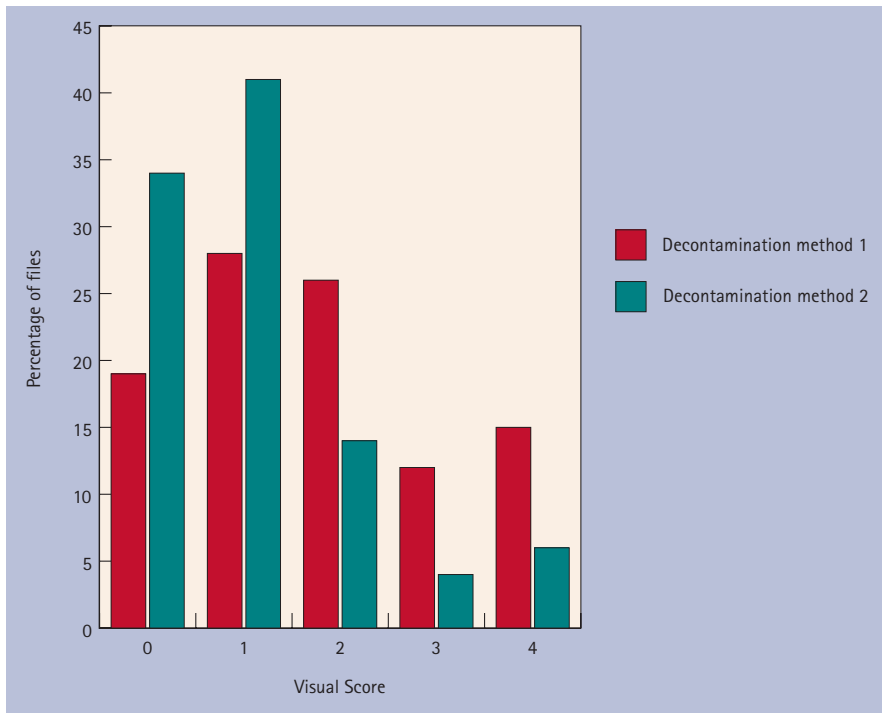

Fig. 2 Visual contamination scores for practices using Decontamination Methods 1 and 2

the samples to be collected prior to the visit. A coding system was used for each practice to maintain anonymity. A questionnaire was administered to each dental practitioner during a practice visit and completed via a face to face interview. The visits were carried out by one investigator who is a dental practitioner (SL). The questionnaire was used to make an assessment of the types of endodontic files used, cross infection control procedures related to endodontic files and methods used to reprocess endodontic files.

At the same visit, 10 endodontic files were collected from each practice and replaced with new stock. The removed files had been used to treat at least one patient and subjected to the routine decontamination procedures for that practice. No attempt was made to identify the number of times each file had been used and reprocessed, since it would be unusual for dental practices to record this information. The files were coded in the same manner as the questionnaire, to maintain anonymity. All files were collected using sterile forceps and stored in sterile containers.

\section{Visual examination}

Ten files from each practice were examined visually for debris by means of a dissecting light microscope (Wild M3Z -Heerbrugg, Switzerland), at magnifications of x16 and x40. A previously described coding system ${ }^{8}$ was employed to quantify residual debris on each file (Table 1). The files were examined by one investigator and were examined blind in relation to the practice and questionnaire.

\section{Kastle-Meyer test}

Following visual examination, the files were then subjected to the Kastle-Meyer test for residual blood contamination. ${ }^{9}$ This well known forensic science test for haemoglobin has been used in previous investigations of residual blood on dental surgery surfaces and equipment. ${ }^{10,11}$ The sensitivity of the test allows detection of blood in dilutions of between 1:50,000 and $1: 100,000$.

A metal tray was used to contain all test materials. Each file to be tested was swabbed on the edge of a folded filter paper. The filter paper was then unfolded and the sample on each paper was tested by placing one drop of Kastle-Meyer reagent on the centre, followed by one drop of isopropyl alcohol and then one drop of hydrogen peroxide (10 volumes). A positive result was shown by development of a vivid pink colour in 20 seconds or less.

The files were tested in groups of five. A new file wiped in bovine blood was used as a positive control and a new, unused file as a negative control. One investigator carried out all tests.

\section{Data analysis}

The questionnaire data and results of both the visual examination and the Kastle-Meyer test were entered into a computer database for subsequent analysis using Minitab (v12.0). Median visual scores were compared using the Mann-Whitney test (for two groups) and the Kruskal Wallis test (when considering more than two groups). The chi-squared test was used to examine for potential associations between method of cleaning and both the visual score and Kastle-Meyer test results.

\section{RESULTS}

\section{Endodontic files}

Of the 25 practices, 19 used stainless steel hand files, four nickel titanium rotary files, one stainless steel in a gyromatic handpiece and one both nickel titanium and stainless steel files.

The majority of practitioners (92\%) changed files when they were bent or damaged. None of the practitioners in the study changed the files after each patient. Eighty-four per cent of the practitioners stated they did not use the files as single use devices because of the increased cost that would be incurred. A small proportion (12\%) did not think that it was necessary to change the files after each patient.

\section{Visual contamination}

Two hundred and fifty files (10 files from each practice) were visually examined prior to performing the Kastle-Meyer test. The results are summarised in Table 1 . These results show that 75\% of all files showed some degree of visual contamination. A photomicrograph of a reprocessed file is shown in Figure 1.

\section{Kastle-Meyer test}

Seventeen (6.8\%) of the 250 files tested positive for blood with the Kastle-Meyer test (Table 2).

\section{Relationship between Kastle-Meyer results and visual contami- nation}

The median visual score of the files that were positive by the Kastle-Meyer test was 2 (range 0-4), compared with a median visual score of 1 (range 0-4) for the Kastle-Meyer negative files. This difference was statistically significant $(p=0.035)$ indicating that the blood contaminated files were visually more extensively contaminated. 


\section{Decontamination of endodontic files}

There was wide variation in the methods used by practices to clean endodontic files. The cleaning process varied from a wipe with an alcohol impregnated cloth (Azowipe ${ }^{\circledR}$ ) to hand scrubbing or use of an ultrasonic bath. The most common method of cleaning was manual cleaning with brushes, and the most common method of sterilisation was in a bench top steam steriliser.

Ten practices used the same method of decontamination, consisting of hand scrubbing the files followed by steam sterilisation in the autoclave (Method 1). A further seven practices employed hand scrubbing, ultrasonic bath and steam sterilisation in the autoclave (Method 2). For the purposes of statistical analysis these two methods of decontamination were examined more closely.

\section{Visual score and method of decontamination}

A Chi-squared test of association between method of decontamination (1 or 2 ) and the visual results showed evidence of association $(\mathrm{p}=0.006)$, with Method 2 being more effective than method 1 (Fig. 2). Examination of median values of visual contamination confirmed this, showing that the median value for Method 1 was 2 (range 0-4) and for Method 2 was 1 (range $0-4)(\mathrm{p}<0.001)$.

\section{Kastle-Meyer test results and method of decontamination}

There was no evidence of a statistically significant association between method of decontamination and the presence or absence of blood ( $\mathrm{p}=0.704)$.

\section{Variations in residual contamination following use of each decontamination method \\ Method 1 - Visual score}

The median visual scores for the 10 practices using decontamination Method 1 ranged from 0.5 to 2.5. A test of equal medians across the 10 practices resulted in a p-value of 0.042 , showing evidence of a difference in visual contamination between practices using manual cleaning followed by steam sterilisation.

\section{Method 2 - Visual scores}

The median visual scores for the seven practices using decontamination Method 2 ranged from $0-2$. The $p$-value of $<0.001$ in a test of equal medians demonstrated significant differences between practices in terms of visual contamination following manual cleaning, ultrasonication and steam sterilisation.

\section{Kastle-Meyer results}

For both decontamination Methods 1 and 2, there was marked variation in the numbers of files with residual blood following use of either method.

\section{DISCUSSION}

The recent Department of Health risk assessment for vCJD and dentistry $^{6}$ categorises dentistry as 'low risk' for potential transmission of vCJD. However, the report recognises that the possibility of infectivity in dental pulpal tissue cannot be ruled out and endodontic files, the instruments coming in direct contact with the pulp, are especially difficult to clean. In particular, the risk assessment assumes that optimal reprocessing standards are observed. The difficulties in cleaning endodontic files, which are highlighted in this paper, are, therefore, a concern.

The number of occasions on which each file had been used was not determined, since in the general dental practices being studied, there were no methods in use for tracing instruments through the decontamination process. However, there may be a cumulative build-up of contaminants on files during multiple episodes of reuse. The heat of sterilisation may also act to fix residual material onto the surface of the file, resulting in a vicious cycle if files are

\begin{tabular}{l} 
Table 1 Visual contamination of files subjected to Kastle-Meyer test \\
\begin{tabular}{lll}
\hline Visual score* & Number of files (\%) \\
\hline 0 & No debris visible at low or high power & $63(25)$ \\
\hline $1 \quad$ Debris found on 1-25\% instrument length & $90(36)$ \\
\hline $2 \quad$ Debris found on $25-50 \%$ instrument length & $50(20)$ \\
\hline $3 \quad$ Debris found on $50-75 \%$ instrument length & $22(9)$ \\
\hline $4 \quad$ Debris found on $>75 \%$ instrument length & $25(10)$ \\
\hline
\end{tabular} \\
*Smith et al. (2002) \\
\hline
\end{tabular}

\begin{tabular}{ll} 
Table 2 Positive Kastle-Meyer results for each practice \\
\hline $\begin{array}{l}\text { Number of blood contaminated } \\
\text { files }\end{array}$ & $\begin{array}{l}\text { Number of practices with blood } \\
\text { contaminated files }\end{array}$ \\
\hline 0 & 13 \\
\hline 1 & 8 \\
\hline 2 & 3 \\
\hline 3 & 1 \\
\hline
\end{tabular}

re-used on multiple occasions. The study reported in this paper supports the contention that endodontic files cannot be reliably cleaned following use. Of interest is the fact that seven practices followed one of the manufacturer's instructions for decontamination (Method 2 - hand scrubbing, ultrasonication and steam sterilisation). These results question the robustness and validity of manufacturer's instructions for the decontamination of endodontic files and the regulatory processes currently in place for reviewing the efficacy of such instructions. Some manufacturers label endodontic files as single-use devices and others have instructions that are incompatible with the commonly used sterilisation cycles in the UK. Data were not collected in the present study on manufacturers' instructions for the particular file used in each practice. However, re-use of devices labelled by the manufacturer as singleuse or failure to follow the manufacturers' instructions would render the practitioner liable to legal action.

Seventy-five per cent of the files examined in this part of the study were visually contaminated after processing. This figure is comparable with that in a previously reported, smaller scale study, using the same scoring system, in which $76 \%$ of files from general practice were visually contaminated. ${ }^{8}$ Many of the files could be seen to be visibly contaminated without use of magnification, yet all files had been accepted by practitioners as ready for re-use.

It is well documented that blood can transmit infection and present a risk to both health care workers and patients. ${ }^{12,13}$ The Kastle-Meyer test for blood deposits produces a well defined end point and has a high degree of sensitivity, but does have a potential for false positives. ${ }^{14}$ Common dental materials have previously been tested to eliminate the potential for false positives from these. Materials tested include calcium hydroxide, gutta percha, zincoxide eugenol cement, zinc polycarboxylate cement, zinc phosphate, composite resin materials, glass ionomer cements and amalgam. ${ }^{11}$ The fact that 17 re-processed endodontic files tested positive for blood is a reminder that thorough cleaning of used instruments is essential if transmission of a wide range of pathogenic microorganisms is to be prevented.

These results demonstrate that endodontic files are routinely contaminated with tissue debris after reprocessing, and as such cannot be excluded as a potential risk of transmitting infectious agents. While the data have shown a significant difference in the effectiveness of the two most common cleaning regimes, it is important to note that there were also significant differences between practices using the same method. This highlights the unreliable and unpredictable nature of the process. At present, the vast majority of practitioners are re-using endodontic files, although the laboratory data presented suggest that they should be 
viewed as single use devices. This raises important issues linked to the funding of endodontic procedures in the health service, which will need to be resolved. However, unless more reliable cleaning methods become available, then the re-processing of endodontic files will remain a procedure of uncertain quality.

1. Miller C H. Infection Control. Dent Clin NAm 1996; 40: 437-456.

2. Head M W, Ritchie $\mathrm{D}$, McLoughlin $\mathrm{V}_{\text {, Ironside J W. Investigation of PrPres in dental }}$ tissues in variant CJD. Br Dent J 2003; 195: 339-343.

3. Glatzel $M$, Abela $E_{1}$ Maissen $M$, Aguzzi A. Extraneural pathologic prion protein in sporadic Creutzfeldt-Jakob Disease. N Engl J Med 2003; 349: 1812-1820.

4. Zobeley E, Flechsig E, Cozzio A, Enari M, Weissmann C. Infectivity of scrapie prions bound to a stainless steel surface. Mol Med 1999; 5: 240-243.

5. Department of Health. Risk assessment for transmission of VCJD via surgical instruments: a modelling approach and numerical scenarios. London: Economics and Operational Research Division, March 2001.

6. Department of Health. Risk assessment for VCJD and dentistry. London: Economics and Operational Research Division, July 2003.
7. Bagg J, Sweeney C P, Roy K M, Sharp T, Smith A J. Cross infection control measures and the treatment of patients at risk of Creutzfeldt Jakob Disease in UK genera dental practice. Br Dent J 2001: 191: 87-90.

8. Smith A, Dickson M, Aitken J, Bagg J. Contaminated dental instruments. J Hosp Infect 2002; 51: 233-235.

9. Glaister J. The Kastle-Meyer test for the detection of blood. Br Med J 1926; 10: 650-652.

10. McColl E, Bagg, J, Winning S. The detection of blood on dental surgery surfaces and equipment following dental hygiene treatment. Br Dent J 1994; 176 65-67.

11. Lowe A H, Bagg J, Burke F J T, Mackenzie D, McHugh S. A study of blood contamination of Siqveland matrix bands. Br Dent J 2001; 192: 43-45.

12. Cleveland J L, Cardo D M. Occupational exposures to human immunodeficiency virus, hepatitis B virus, and hepatitis C virus: risk, prevention, and management. Dent Clin N Am 2003; 47: 681-696

13. Sundkvist $T$, Hamilton $G$ R, Rimmer D, Evans B G, Teo C G. Fatal outcome of transmission of hepatitis $B$ from an e antigen negative surgeon. Commun Dis Public Health 1998; 1: 48-50.

14. Cox M. A study of the sensitivity and specificity of four presumptive tests for blood. $J$ Forensic Sci 1991; 36: 1503-1511. 